Cite this: Phys. Chem. Chem. Phys., 2013 15, 20115

Received 19th August 2013,

Accepted 1st October 2013

DOI: $10.1039 / c 3 c p 53522 c$

www.rsc.org/pccp

\title{
Surface-charge distribution on a dielectric sphere due to an external point charge: examples of $C_{60}$ and $C_{240}$ fullerenes
}

\author{
Gerardo Raggi, Anthony J. Stace and Elena Bichoutskaia*
}

\begin{abstract}
An analytical solution for the distribution of surface charge on a dielectric sphere due to the presence of an external point charge is presented. This solution describes how charge on the surface of the sphere is polarised in the electric field into regions of negative and positive charge. The polarisation effect (distribution of surface charge) generally varies with the separation between the sphere and the charge, and it is particularly significant at very short separations. Results obtained from the classical electrostatic model are in qualitative agreement with density functional theory calculations of charge separation in $C_{60}$ and $C_{240}$ fullerenes in the presence of an external point charge. This suggests that, from an electrostatic point of view, in the static electric field of external charges these molecules exhibit dielectric behaviour.
\end{abstract}

\section{Introduction}

An understanding of the electrostatic properties of dielectric materials has important consequences for many industrial processes and procedures. Prime examples where electrostatic effects can have a significant influence on particle behaviour include removal of fine particles and coal dust from the atmosphere in power stations, ${ }^{1}$ particle charging and deposition during powder coating, ${ }^{2}$ and the dynamic behaviour of particles in dry powder inhalers. ${ }^{3}$

In an earlier publication, ${ }^{4}$ an analytical solution to the classical problem describing how charged particles of dielectric materials interact with one another was presented. Using this solution it has been possible to explain why like-charged particles of dielectric materials could attract one another, and to show that such attraction arises from a mutual polarisation of bound charge on the interacting particles due to an electric field generated by the particles. ${ }^{5}$ To obtain an analytical solution for the force between two charged particles, the position-dependent electrostatic potential was expanded as a Legendre polynomial series, and appropriate boundary conditions were introduced. Since the ability of an external field to polarise materials is an essential component of the electrostatic interaction between charged dielectric particles, ${ }^{6}$ it is important to test the validity of the adopted electrostatic approach, especially at the nano-scale, by means of comparison with relevant quantum chemical calculations.

Department of Physical and Theoretical Chemistry, School of Chemistry, University of Nottingham, University Park, Nottingham NG7 2RD, UK. E-mail: Elena.Bichoutskaia@nottingham.ac.uk
The counterpart problem of a conducting particle in the presence of an external point charge was first proposed by Kelvin in $1845,{ }^{7}$ which eventually led to the development of the image charge theory. ${ }^{6}$ In this paper, the analytical solution ${ }^{4}$ is adapted to the problem of a point charge placed outside a dielectric particle. The results obtained by the classical electrostatic analysis are compared with the distribution of surface charge density in $\mathrm{C}_{60}$ and $\mathrm{C}_{240}$ fullerenes computed using density functional theory (DFT). For each example, the influence of an external point charge on partial atomic charges on the fullerene molecule is investigated and compared with the polarisation charge induced on the surface of the dielectric sphere.

This study contributes to an on-going debate ${ }^{8-10}$ on whether or not a single $\mathrm{C}_{60}$ molecule can be treated as a dielectric particle. The defining characteristic of a metal, or more generally a conductor, is the presence of a finite density of states (DOS) at the Fermi level. Both photoemission experiments ${ }^{11}$ and in particular scanning tunnelling spectroscopy, ${ }^{12}$ where a fullerene is effectively incorporated into an electric circuit, find no evidence for the presence of a finite DOS at the Fermi level both for a fullerite crystal (or thin fullerene film) and individual $\mathrm{C}_{60}$ molecules. If the $\mathrm{C}_{60}$ molecule was a purely metallic particle one would expect to see metallic screening of the core-hole created in photoemission from the $\mathrm{C} 1$ s core level. This type of screening gives rise to a very distinctive line-shape known as the Doniach-Sunjic line profile, ${ }^{13}$ which is essentially a measure of the metallic DOS at the Fermi level. The photoemission spectrum of both highly oriented pyrolytic graphite and glassy carbon shows an asymmetric core-level peak-shape due to the collective screening of the core hole created by the 
photoemission process (see Fig. 3 of ref. 14). However for the $\mathrm{C}_{60}$ molecules, the core-level emission is almost symmetric along the energy axis. ${ }^{14,15}$ This suggests that the $\mathrm{C}_{60}$ molecule behaves like a dielectric particle under the influence of a static electric field.

\section{Distribution of polarisation charge on the spherical interface due to the presence of external point charge}

The distribution of charge on the spherical interface induced by the presence of an external point charge is derived using a Legendre polynomial expansion. A point charge of magnitude $q$ is located outside a dielectric sphere of charge $Q$ and radius $a$ at a distance $h=|\boldsymbol{h}|$ from the centre of the sphere. Fig. 1 illustrates the geometry of the dielectric sphere-point charge interaction. The dielectric constant, $k$, of the sphere is dimensionless as it is defined as the permittivity relative to that of vacuum, i.e. $k=\varepsilon / \varepsilon_{0}$, where $\varepsilon_{0}=8.8541878176 \times 10^{-12} \mathrm{~F} \mathrm{~m}^{-1}$.

The electrostatic potential $\Phi(\boldsymbol{r})$ at any point in space $\boldsymbol{r}$ is found by solving the Poisson differential equation

$$
\nabla^{2} \Phi(\boldsymbol{r})=-\frac{\sigma(\boldsymbol{r})}{\varepsilon_{0}}
$$

where $\sigma(\boldsymbol{r})$ is the charge density. The Gauss potential $\Phi(\boldsymbol{r})$ is defined as a sum of the contributions from the sphere (first term) and the external point charge (second term)

$$
\Phi(\boldsymbol{r})=K \int \frac{\sigma(\tau) \mathrm{d} \tau}{|\boldsymbol{r}-\boldsymbol{a}(\tau)|}+K \frac{q}{|\boldsymbol{r}-\boldsymbol{h}|}
$$

where $K=\frac{1}{4 \pi \varepsilon_{0}}$ is a proportionality coefficient. In the first term of eqn (2) there is a singularity on the surface of the sphere where $|\boldsymbol{r}-\boldsymbol{a}| \equiv 0$, which results in two separate expressions inside and outside the sphere. For $\boldsymbol{r}<\boldsymbol{a}$ (inside the sphere), this term takes the form ${ }^{4}$

$$
K \int \frac{\sigma(\tau) \mathrm{d} \tau}{|\boldsymbol{r}-\boldsymbol{a}(\tau)|}=\sum_{l=0}^{\infty} A_{l} \frac{r^{l}}{a^{2 l+1}} P_{l}(\cos \beta)
$$

and for $\boldsymbol{r}>\boldsymbol{a}$ (outside the sphere) it has the form

$$
K \int \frac{\sigma(\tau) \mathrm{d} \tau}{|\boldsymbol{r}-\boldsymbol{a}(\tau)|}=\sum_{l=0}^{\infty} A_{l} \frac{1}{r^{l+1}} P_{l}(\cos \beta)
$$

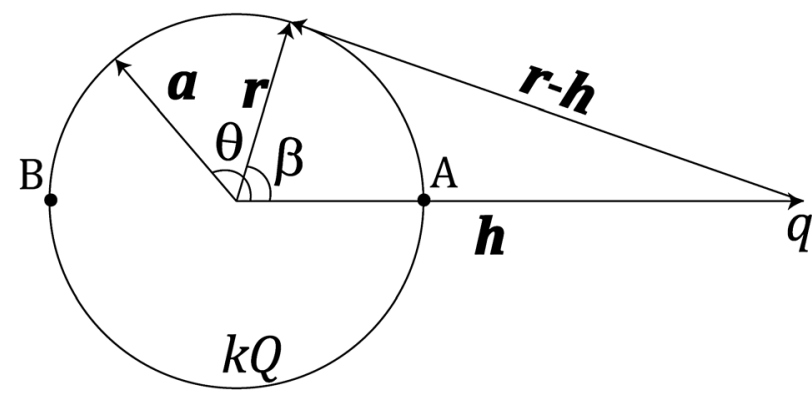

Fig. 1 Geometric representation of the sphere-point charge interaction. where $P_{l}(\cos \beta)$ is the Legendre polynomial, and

$$
A_{l}=2 \pi K a^{l+2} \int_{0}^{\pi} \sin \theta \mathrm{d} \theta \sigma(\theta) P_{l}(\cos \theta)
$$

assuming spherical polar coordinates $(a, \theta, \varphi)$ for vector $\boldsymbol{a}$ and $\left(r, \beta, \varphi_{1}\right)$ for vector $\boldsymbol{r}$ (see Fig. 1).

We impose the boundary conditions, ${ }^{4}$ which state that the normal components of the dielectric displacement field $\mathbf{D}$ and the tangential components of the electric field $\mathbf{E}$ satisfy the following equations

$$
\begin{aligned}
& \left(\left.\mathbf{D}\right|_{r=a^{+}}-\left.\mathbf{D}\right|_{r=a^{-}}\right) \times \mathbf{n}=0 \\
& \left(\left.\mathbf{E}\right|_{r=a^{+}}-\left.\mathbf{E}\right|_{r=a^{-}}\right) \times \mathbf{n}=0
\end{aligned}
$$

where $a^{+}$and $a^{-}$refer to a radial position just outside and inside the sphere, respectively; $\mathbf{n}$ is a unit vector normal to the surface and directed outwards from the sphere surface.

The total charge, $\sigma$, on the surface of the sphere is defined as a sum of a uniformly distributed free charge $Q$ and a bound polarisation charge $\sigma_{\text {surf. }}^{\text {pol }}$ Inside the sphere, the volume density of polarization charge is zero since $\sigma_{\mathrm{vol}}^{\mathrm{pol}}=(1-k) \varepsilon_{0} \nabla \cdot \boldsymbol{E}=-\nabla \cdot \boldsymbol{P}=0$ (except at $\boldsymbol{r}=\boldsymbol{h}$ ). At the interface, therefore, the normal component of the electric field is discontinuous due to the presence of a permanent surface charge ${ }^{4}$

$$
\frac{\sigma}{\varepsilon_{0}}=\left.\nabla \Phi\right|_{r=a^{-}}-\left.\nabla \Phi\right|_{r=a^{+}}
$$

noting that $\boldsymbol{E}=-\nabla \Phi$.

Expansions (3) can be used to express the boundary condition (6) using Legendre polynomials to give an expression for the polarisation surface charge density

$$
\sigma_{\text {surf }}^{\mathrm{pol}}(\cos \beta)=\varepsilon_{0} \sum_{l=0}^{\infty} A_{l} \frac{2 l+1}{a^{l+2}} P_{l}(\cos \beta)
$$

Using the matrix relationship given by eqn (16) of ref. 4 to yield the coefficient $A_{l}$, the final expression for $\sigma_{\text {surf }}^{\text {pol }}$ can be expressed as

$$
\sigma_{\text {surf }}^{\mathrm{pol}}(\cos \beta)=\frac{q}{4 \pi h^{2}} \sum_{l=1}^{\infty}(2 l+1) l \frac{(1-k)}{(1+k) l+1}\left(\frac{a}{h}\right)^{l-1} P_{l}(\cos \beta)
$$

Eqn (8) describes how the density of surface charge on the dielectric sphere is influenced by the presence of a point charge $q$ located at a distance $h$ from the centre of the sphere. This analytical expression agrees with the treatment presented in ref. 16 for a study of the effects of an image charge for the problem of an individual micro-ion interacting with a dielectric sphere.

Fig. 2 shows the surface charge density distribution, obtained using eqn (8), on a neutral sphere $(Q=0)$ of radius $a=3.8 \AA$ and dielectric constant $k=3.45$, which has the dimensions ${ }^{17}$ and dielectric properties ${ }^{18}$ of the $\mathrm{C}_{60}$ molecule. The surface charge density for each value of the angle $\beta$ has been weighted by an element of surface area, and it is presented in Fig. 2 in the form of $2 \pi a^{2} \sin (\beta) \sigma(\cos \beta) .{ }^{5}$ The curves in Fig. 2 show the distribution of the surface charge as a function of the angle $\beta$ ( $x$-axis) and 


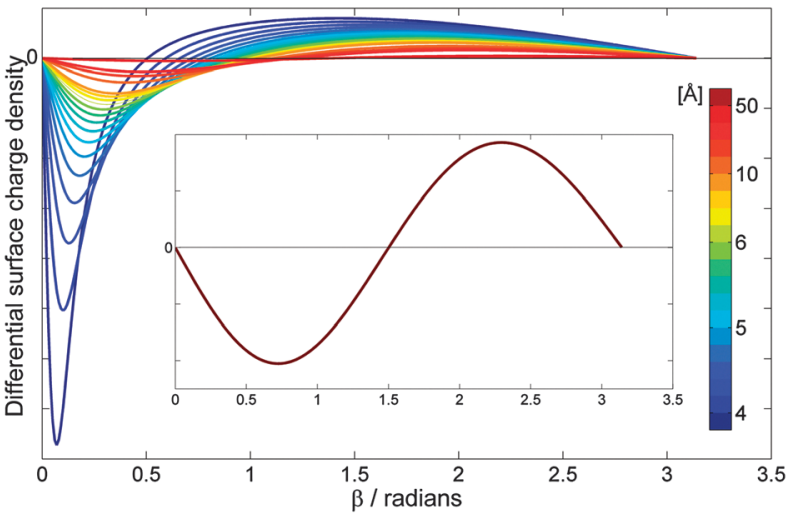

Fig. 2 Differential surface charge density, calculated as $2 \pi a^{2} \sin (\beta) \sigma(\cos \beta)$ using eqn (8), on a sphere representing the dimensions and dielectric properties of the $C_{60}$ molecule: $a=3.8 \AA k=3.45, Q=0$. The point charge has a magnitude of $q=+15$ e. The colour labeling is used to represent the separation, $h$, between a point charge and the centre of the sphere, which varies with a step of $0.2 \AA$ in the distance range of $4.2 \AA$ (dark blue) to $7 \AA$ (green), thereafter $h$ is taken to be $8,9,10,15,20$, and $50 \AA$ (deep red). Inset shows the surface charge density distribution at $h=50 \AA$

the separation distance $h$ (colour labels) between the sphere and a positive point charge $q=+15 e$. It can be seen from Fig. 2 that the surface charge density of a neutral sphere is polarized in the presence of external point charge, and the density distribution varies significantly as a function of sphere-point charge separation. The polarisation effects become more profound as the point charge is brought closer to the sphere reaching a maximum near the contact point $(h=a)$. It turns out that a point charge continues to exert a significant influence on the neutral particle up to a separation distance of $50 \AA$ (inset of Fig. 2). At larger separation distances $(h>50 \AA)$, the surface charge becomes uniformly distributed over the surface, and effectively becomes independent of $h$. Therefore, previous treatments ${ }^{19-24}$ in which a uniform (or non-uniform but independent of the separation distance) distribution of surface charge is assumed are only valid at large separations between a polarizable particle and external charge.

As shown in Fig. 2 the value of the differential surface density is zero for $\beta=0$ and $\beta=\pi$ as these angles correspond to a density at a point (points A and B in Fig. 1). The lobe of negative charge describes the charge density on the surface area close to the external positive charge, and the lobe of positive charge accounts for the density smeared towards $\beta=\pi$. It can be seen that the surface charge density is polarised more towards the external charge, and the effect is more pronounced for shorter separations, typically for $h$ less than $7 \AA$. At $h=50 \AA$ (inset in Fig. 2), the charge is separated in such a way that the negative charge occupies the entire hemisphere close to the external charge, whereas positive charge is located in the hemisphere defined by $\beta \in\left(90^{\circ}, 270^{\circ}\right)$. Upon a further increase in the charge-sphere separation $h$, the induced polarisation charge approaches zero (no polarisation of the sphere). This charge polarisation behaviour is in close agreement with that described in ref. 5 for the interaction of neutral $\mathrm{C}_{60}$ with $\mathrm{C}_{60}{ }^{2+}$.
The above classical electrostatic analysis of the surface charge density distribution on a dielectric sphere due to the presence of an external point charge can be validated with quantum chemical calculations of partial atomic charges in $\mathrm{C}_{60}$ and $\mathrm{C}_{240}$ fullerenes as described in the next section.

\section{Quantum chemical description of the charge density in $C_{60}$ and $C_{240}$ fullerenes}

In this section the classical electrostatic model is compared with results obtained at the $\mathrm{B}^{2} \mathrm{LYP}^{25,26} / 6-311 \mathrm{G}^{* 27}$ DFT level of theory, as implemented in the $Q$-Chem quantum chemistry package ${ }^{28}$ for the distribution of charge in the $\mathrm{C}_{60}$ and $\mathrm{C}_{240}$ molecules in the presence of an external point charge. Partial atomic charges on the carbon atoms in $\mathrm{C}_{60}$ and $\mathrm{C}_{240}$ fullerenes have been calculated using the Mulliken population analysis ${ }^{29}$ and subsequently compared with the surface charge density predicted by eqn (8).

Fig. 3 depicts the distribution of partial atomic charges on a neutral $\mathrm{C}_{60}$ molecule, as predicted by the Mulliken population analysis, at three separation distances between the point charge and the centre of $\mathrm{C}_{60}$. Mulliken analysis ${ }^{29}$ is the oldest and simplest population analysis method, and it is widely accepted that the absolute magnitude of the atomic charges is not accurate as their values display a high degree of sensitivity to the atomic basis set with which they were calculated. ${ }^{30}$ However, the location of charge within the molecule is predicted reliably provided a consistent basis set is used. Three values of the point charge- $\mathrm{C}_{60}$ separation distance have been considered, namely $h=5 \AA$ (Fig. 3a), $h=10 \AA$ (Fig. 3b) and $h=50 \AA$ (Fig. 3c), showing an increasing de-localization of negative charge on fullerene, depicted in green in Fig. 3, as the point charge, located to the right of the fullerene cage (not shown in Fig. 3) moves away from the molecule. It can be clearly seen that at $h=50 \AA$ the negative charge spreads over the entire hemisphere and the charge density on the neutral $\mathrm{C}_{60}$ remains polarized, which is consistent with the classical electrostatic analysis presented in the inset of Fig. 2. The point charge with the value of $q=+15 e$ continues to exert a strong influence on the neutral fullerene cage even at distances greater than $50 \AA$.

For a comparison with the continuous charge density distribution derived from the classical electrostatic model (Fig. 2) a Gaussian distribution function has been used to represent the
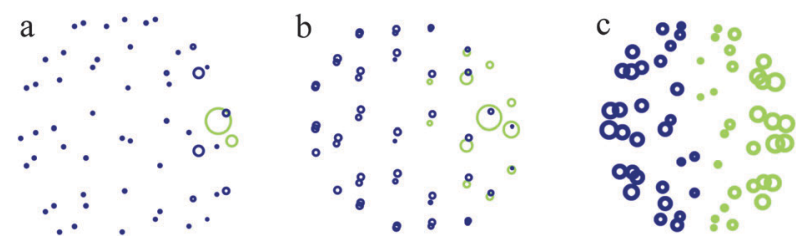

Fig. 3 Distribution of partial atomic charges on the $C_{60}$ molecule at three values of separation distance between the centre of $C_{60}$ and the external point charge of $q=+15$ e located to the right of the fullerene cage (not shown): (a) $5 \AA$, (b) $10 \AA$, and (c) $50 \AA$. Blue circles show positive charge residing on carbon atoms, and green circles depict negative charge. The size of each circle has been renormalized to convey the degree of polarization rather than its magnitude. 


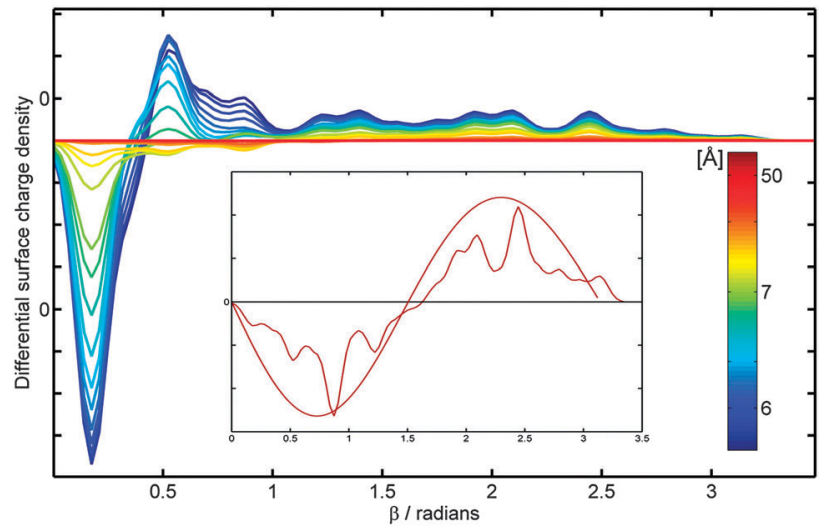

Fig. $4 C_{60}$ surface charge density, obtained from the Mulliken population analysis, in the presence of the external point charge of $q=+15 \mathrm{e}$. The colour labeling is used to represent the separation, $h$, between a point charge and the centre of $C_{60}$, which varies with a step of $0.2 \AA$ in the distance range of $5 \AA$ (dark blue) to $7 \AA$ (green), thereafter $h$ is taken to be $8,9,10,15,20$, and $50 \AA$ (deep red); $\Delta \beta=10^{\circ}$ and $n=2$. Inset shows the surface charge density distribution at $h=50 \AA$ compared to the analytical solution shown in the inset of Fig. 2 .

computed net atomic charges, which has an effect of smearing the charge density across the surface of the molecule. Eighteen slices of the surface of $\mathrm{C}_{60}$ with the same width (defined by a fixed incremental angle $\Delta \beta=10^{\circ}$ ) have been considered. For each slice a normal distribution function is used to smear the charge determined by the Mulliken population analysis. The index $n$ is used to define the number of slices over which each Gaussian function extends. In Fig. 4, the partial charge is distributed only within each slice $(n=2)$, thus showing not only a clear polarization of the negative and positive charges for small values of angle $\beta$ but also small peaks corresponding to residual positive charges on atoms further away from the external charge.

For the larger neutral $\mathrm{C}_{240}$ molecule whose radius is taken to be $7.3 \AA$, the distribution of partial atomic charges is presented in Fig. 5 for two separation distances of $h=9 \AA$ (Fig. 5a) and $h=20 \AA$ (Fig. 5b). The corresponding charge density distribution for the optimized geometry of $\mathrm{C}_{240}$ and $\Delta \beta=10^{\circ}$ is shown in Fig. 6 for $n=2$. These results are qualitatively similar to the case of $\mathrm{C}_{60}$ presented in Fig. 3 and 4.
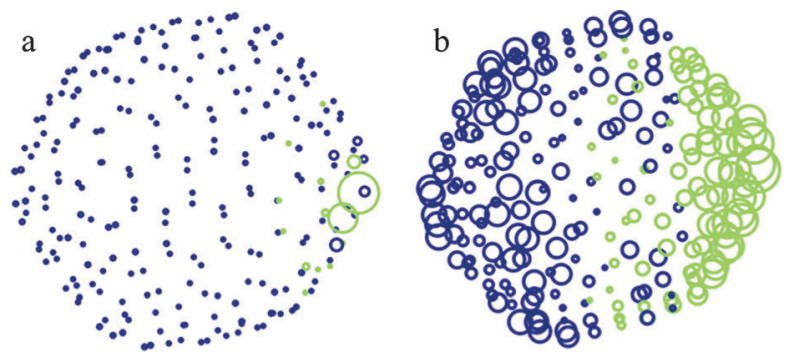

Fig. 5 Distribution of partial atomic charges on the $C_{240}$ molecule at two values of separation distance between the centre of $C_{240}$ and the external point charge of $q=+15$ e located to the right of the fullerene cage (not shown): (a) $9 \AA$ and (b) $20 \AA$. Further details can be found in the caption to Fig. 3.

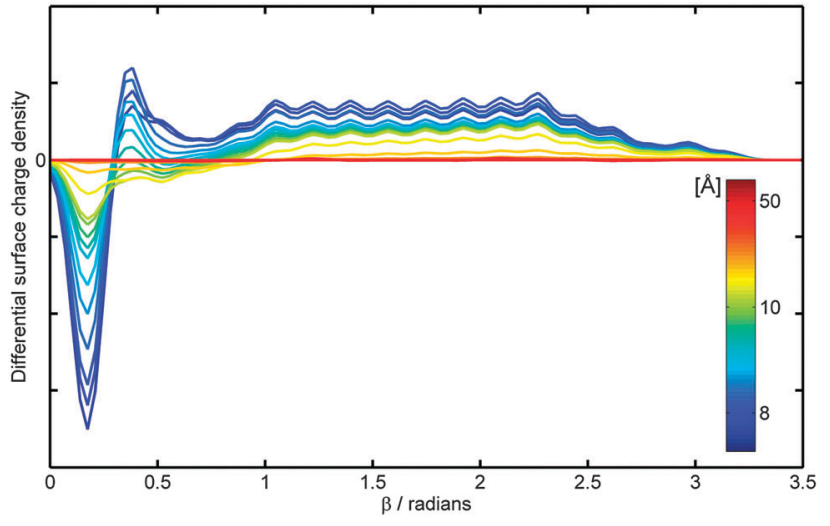

Fig. $6 C_{240}$ surface charge density, obtained from the Mulliken population analysis, in the presence of the external point charge of $q=+15 e$. The colour labeling is used to represent the separation, $h$, between a point charge and the centre of $C_{240}$, which varies with a step of $0.2 \AA$ in the distance range of $8 \AA$ (dark blue) to $10 \AA$ (green), thereafter $h$ is taken to be 12,20 and $50 \AA$ (deep red); $\Delta \beta=10^{\circ}$ and $n=2$.

\section{Conclusions}

The recently developed classical model ${ }^{4}$ to describe electrostatic interactions between charged spheres of a dielectric material requires the material to be susceptible to polarisation in the presence of an external electric field. In order to test this assumption, the model has been adapted to the particular problem of a dielectric sphere interacting with a point charge. For this problem an expression is given for the displacement of bound charge density on the sphere as a function of separation between a point charge and the centre of a sphere. Comparisons have been made between the classical electrostatic results and those derived from a DFT study of the bound surface charge density on the dielectric molecular spheres $\mathrm{C}_{60}$ and $\mathrm{C}_{240}$. By smearing the calculated partial atomic charges it is shown that there are marked similarities between these two approaches. This suggests that the classical electrostatic picture used to describe the behaviour of dielectric materials in the presence of an electric field is appropriate for the treatment of small particles. Furthermore, the proposed model might have implications in experiments involving the fullerene multiple-charged ion collisions. ${ }^{31,32}$ In experiments concerned with charge-transfer processes the insight into polarization of fullerenes is the first step in the understanding of their fission mechanism.

\section{Acknowledgements}

We thank Prof. P. Moriarty for fruitful discussions on conducting properties of individual fullerene cages. GR acknowledges the CONACYT Mexico Scholarship for PhD funding (Reg. 215343). EB acknowledges financial support through an ERC Starting Grant, an EPSRC Career Acceleration Fellowship, and a New Directions for EPSRC Research Leaders Award (EP/G005060).

\section{References}

1 A. Jaworek, W. Balachandran, A. Krupa, J. Kulon and M. Lackowski, Environ. Sci. Technol., 2006, 40, 6197. 
2 X. Meng, J. J. Zhu and H. Zhang, J. Phys. D: Appl. Phys., 2009, 42, 065201.

3 A. Hickey, H. Mansour and M. Telko, J. Pharm. Sci., 2007, 96, 1302.

4 E. Bichoutskaia, A. L. Boatwright, A. Khachatourian and A. J. Stace, J. Chem. Phys., 2010, 133, 024105.

5 A. J. Stace, A. L. Boatwright, A. Khachatourian and E. Bichoutskaia, J. Colloid Interface Sci., 2011, 354, 417.

6 J. D. Jackson, Classical Electrodynamics, Wiley, New York, 1999.

7 W. Thomson (Lord Kelvin), J. Math. Pure. Appl., 1845, 10, 364.

8 A. J. Stace and E. Bichoutskaia, Phys. Chem. Chem. Phys., 2011, 13, 18339.

9 H. Zettergren, O. B. Forsberg and H. Cederquist, Phys. Chem. Chem. Phys., 2012, 14, 16360.

10 A. J. Stace and E. Bichoutskaia, Phys. Chem. Chem. Phys., 2012, 14, 16771.

11 P. J. Benning, J. L. Martins, J. H. Weaver, L. P. F. Chibante and R. E. Smalley, Science, 1991, 252, 1417.

12 J. L. Wragg, J. E. Chamberlain, H. W. White, W. Kratschmer and D. R. Huffman, Nature, 1990, 348, 623.

13 S. Doniach and M. Sunjic, J. Phys. C: Solid State Phys., 1970, 3, 285.

14 J. A. Leiro, M. H. Heinonen, T. Laiho and I. G. Batirev, J. Electron Spectrosc. Relat. Phenom., 2003, 128, 205.

15 E. Rotenberg, C. Enkvist, P. A. Brühwiler, A. J. Maxwell and N. Mårtensson, Phys. Rev. B: Condens. Matter, 1996, 54, R5279.

16 R. Messina, J. Chem. Phys., 2002, 117, 11062.

17 H. Zettergren, J. Jensen, H. T. Schmidt and H. Cederquist, Eur. Phys. J. D, 2004, 29, 63.

18 M. Nakamuraand and P. Hervieux, Chem. Phys. Lett., 2006, 428, 138.

19 H. Ohshima, J. Colloid Interface Sci., 1995, 170, 432.

20 X. Chu and D. T. Wasan, J. Colloid Interface Sci., 1996, 184, 268.
21 G. Iversen, Y. I. Kharkats and J. Ulstrup, Mol. Phys., 1998, 94, 297.

22 T. P. Doerr and Y. K. Yu, Phys. Rev. E: Stat., Nonlinear, Soft Matter Phys., 2006, 73, 061902.

23 Z. J. Lian and H. R. Ma, J. Chem. Phys., 2007, 127, 104507.

24 H. Ohshima, J. Colloid Interface Sci., 2008, 328, 3.

25 A. D. Becke, J. Chem. Phys., 1993, 98, 5648.

26 C. Lee, W. Yang and R. G. Parr, Phys. Rev. B: Condens. Matter Mater. Phys., 1988, 37, 785.

27 R. Krishnan, J. S. Binkley, R. Seeger and J. A. Pople, J. Chem. Phys., 1980, 72, 650.

28 Y. Shao, L. F. Molnar, Y. Jung, J. Kussmann, C. Ochsenfeld, S. T. Brown, A. T. B. Gilbert, L. V. Slipchenko, S. V. Levchenko, D. P. O’Neill, R. A. Di Stasio Jr, R. C. Lochan, T. Wang, G. J. O. Beran, N. A. Besley, J. M. Herbert, C. Y. Lin, T. V. Voorhis, S.-H. Chien, A. Sodt, R. P. Steele, V. A. Rassolov, P. E. Maslen, P. P. Korambath, R. D. Adamson, B. Austin, J. Baker, E. F. C. Byrd, H. Dachsel, R. J. Doerksen, A. Dreuw, B. D. Dunietz, A. D. Dutoi, T. R. Furlani, S. R. Gwaltney, A. Heyden, S. Hirata, C.-P. Hsu, G. Kedziora, R. Z. Khalliulin, P. Klunzinger, A. M. Lee, M. S. Lee, W. Liang, I. Lotan, N. Nair, B. Peters, E. I. Proynov, P. A. Pieniazek, Y. M. Rhee, J. Ritchie, E. Rosta, C. D. Sherrill, A. C. Simmonett, J. E. Subotnik, H. L. Woodcock III, W. Zhang, A. T. Bell, A. K. Chakraborty, D. M. Chipman, F. J. Keil, A. Warshel, W. J. Hehre, H. F. Schaefer III, J. Kong, A. I. Krylov, P. M. W. Gill and M. Head-Gordon, Phys. Chem. Chem. Phys., 2006, 8, 3172.

29 R. S. Mulliken, J. Chem. Phys., 1955, 23, 1833.

30 E. R. Davidson and S. Chakravorty, Theor. Chim. Acta, 1992, 83, 319.

31 N. Haag, Z. Berényi, P. Reinhed, D. Fischer, M. Gudmundsson, H. A. B. Johansson, H. T. Schmidt and H. Cederquist, Phys. Rev. A: At., Mol., Opt. Phys., 2008, 78, 043201.

32 J. Jensen, H. Zettergren, H. T. Schmidt, H. Cederquist, S. Tomita, S. B. Nielsen, J. Rangama, P. Hvelplund, B. Manil and B. A. Huber, Phys. Rev. A: At., Mol., Opt. Phys., 2004, 69, 053203. 